\title{
Association of cellular localization of glycogen synthase kinase $3 B$ in the digestive tract with cancer development
}

\author{
HIROSHI HIRAKAWA ${ }^{1}$, TOSHIYUKI NAKAYAMA ${ }^{1}$, KENICHIRO SHIBATA $^{1,2}$ and ICHIRO SEKINE $^{1}$ \\ ${ }^{1}$ Department of Tumor and Diagnostic Pathology, Atomic Bomb Disease Institute, \\ Nagasaki University Graduate School of Biomedical Sciences, 1-12-4 Sakamoto, Nagasaki 852-8523; \\ ${ }^{2}$ Division of Surgical Oncology, Department of Translational Medical Sciences, Nagasaki University \\ Graduate School of Biomedical Sciences, 1-7-1 Sakamoto, Nagasaki 852-8501, Japan
}

Received February 16, 2009; Accepted May 6, 2009

DOI: 10.3892/or_00000460

\begin{abstract}
Glycogen synthase kinase $3 \beta(G S K-3 \beta)$ is a multifunctional serine/threonine kinase involved in several signaling pathways. Recently, we reported the polarized localization of GSK-3ß on the apical membrane of normal colon epithelium. To investigate the functions of this molecule, we studied stomach and colorectal cancer tissues. In normal simple columnar epithelium, GSK-3ß was localized with tight junction-associated protein $\mathrm{ZO}-1$ in a single line at the apical cell border. GSK-3ß and ZO-1 were localized in the apical regions of tubular adenocarcinoma, similar to their localization in normal epithelium; however, their localization was different at the invasive front of the cancer and was found to be associated with lymphatic invasion. In signet-ring cell carcinoma of the stomach, the expression of these proteins was reduced and dot-like expression was observed in each cell of the signet-ring cell carcinoma. We speculated that GSK-3ß is involved in glandular structure formation and that the nonapical localization of membrane-localized GSK-3ß in tubular adenocarcinoma is associated with cancer development.
\end{abstract}

\section{Introduction}

The serine/threonine kinase glycogen synthase kinase $3 \beta$ (GSK-3ß) was first identified as a component of a metabolic pathway involved in the regulation of glycogen synthesis (1). Thereafter, GSK-3ß has been reported to regulate various physiological responses in mammalian cells, including protein synthesis, gene expression, subcellular localization of proteins and protein degradation via phosphorylation of various substrates (2-4). GSK-3ß plays a central role in the Wnt path-

Correspondence to: Dr Hiroshi Hirakawa, Department of Tumor and Diagnostic Pathology, Atomic Bomb Disease Institute, Nagasaki University Graduate School of Biomedical Sciences, 1-12-4 Sakamoto, Nagasaki 852-8523, Japan

E-mail: h-hira@nagasaki-u.ac.jpc

Key words: cellular localization, glycogen synthase kinase 3ß, immunohistochemistry way (5) and the activation of the Wnt signaling pathway $(6,7)$ or the dysfunction of the so-called $\beta$-catenin destruction complex has been reported in colorectal neoplasm (8-10). The activation of the Wnt signaling pathway or abnormalities/ deviations in the $\beta$-catenin degradation process results in the accumulation of $\beta$-catenin in the nucleus, where it functions as a transcriptional regulator by interacting with $\mathrm{T}$-cell transcription factor (TCF) to activate Wnt-specific target (5). In the cytoplasm, GSK-3ß forms part of the $\beta$-catenin destruction complex, which regulates the level of $\beta$-catenin by subjected it to ubiquitin-mediated proteasomal degradation (11). Recently, we reported that GSK-3B exhibited polarized localization on the apical membrane of normal colon epithelium and aberrant distribution in colorectal cancer (12). In this study, we investigated the role of the apically localized GSK-3ß in normal gut epithelium and in cancer.

\section{Materials and methods}

Antibodies. Mouse monoclonal anti-GSK-3ß (1:100; BD Bioscience, San Jose, USA), anti- $\beta$-catenin (1:250; BD Bioscience), and anti-E-cadherin (1:250; Dako, Glostrup, Denmark) were used for the immunodetection of proteins. In addition, polyclonal rabbit anti-ß-catenin (1:250; Santa Cruz Biotechnology, Santa Cruz, USA), anti-phosphorylated anti-GSK-3ß (Tyr 216) (1:100; Signalway Antibody, Pearland, USA), anti-ZO-1 (1:250; Lifespan Biosciences, Seattle, USA), and anti-adenomatosis polyposis coli (APC) (1:100; C-terminal; Santa Cruz Biotechnology) antibodies were used.

Immunohistochemical analysis. We randomly chose samples from surgical specimens that were subjected to pathological diagnoses in our institution from 2005 to 2007. Eight colon adenomatous polyp, 40 colorectal cancer and 10 stomach cancer specimens were used in this study. All investigations were approved by our Institutional Review Board and informed consent was obtained from all the participants. We prepared $4-\mu \mathrm{m}$-thick sections from formalin-fixed, paraffin-embedded tissue blocks and subjected the sections to immunohistochemical analyses with an avidin-biotin complex detection system (Vectastain; Vector Laboratories, Burlingame, USA). To facilitate antigen retrieval, the sections were incubated in 

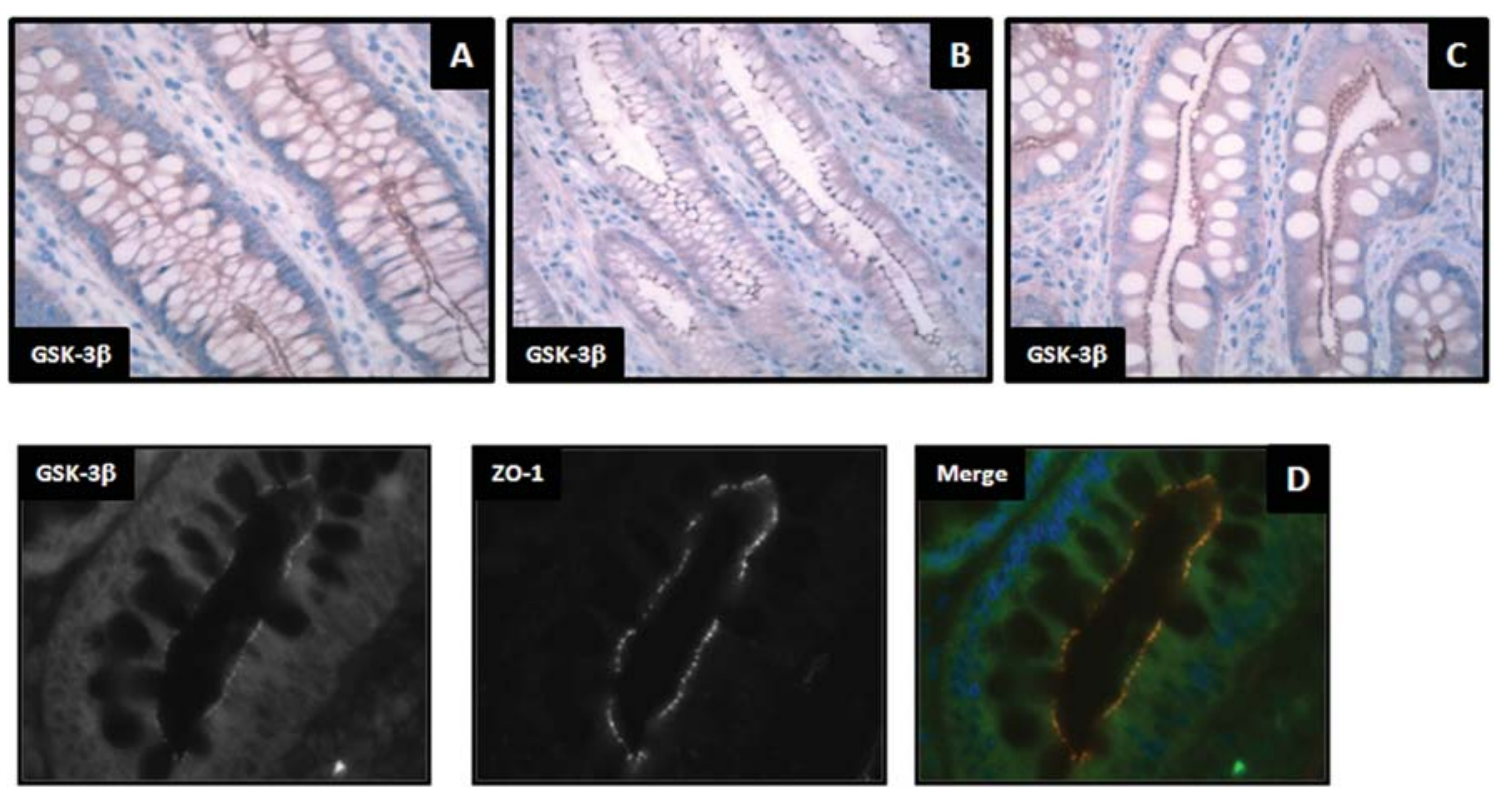

Figure 1. Cellular distribution of GSK-3ß in normal epithelium. (A) Normal epithelium of the colon. (B) Intestinal metaplasia of the stomach epithelium. (C) Normal epithelium of the stomach. (A-C) Immunoreactivity of GSK-3B detected on the apical side of the gut epithelium. (D) Double-immunofluorescence analysis showing the co-localization of GSK-3ß and ZO-1 on the apical side of normal colon epithelium.

Tris-ethylenediaminetetraacetic acid (EDTA) buffer ( $\mathrm{pH} 9.0)$ at $121^{\circ} \mathrm{C}$ for $15 \mathrm{~min}$. Images were captured with a Nikon Digital Sight Fil camera (Nikon, Tokyo, Japan). The immunoreaction for E-cadherin was visualized with an SG substrate kit (Vector Laboratories), which is a proprietary peroxidase substrate that yields a gray color. A diaminobenzidine (DAB) kit (Histofine; Nichirei, Tokyo, Japan) (brown) was used for the detection of B-catenin, GSK-3ß and phosphorylated GSK-3ß (Tyr 216). The control sections were incubated with isotype-matched immunoglobulin (Ig) G.

Immunofluorescence analysis. After incubation with the primary antibodies, the sections were incubated with secondary antibodies (Alexa 488-conjugated affinipure goat anti-mouse IgG and Alexa 555-conjugated affinipure goat anti-rabbit IgG; Molecular Probes, Eugene, USA). Slides were then mounted with Vectashield mounting medium (Vector Laboratories) containing 4,6-diamidino-2-phenylindole (DAPI) for nuclear counterstaining. The fluorescence images were examined using an MD2500 microscope (Leica Microsystems, Tokyo, Japan) with a x40 objective lens. The images were captured with a Leica DC500 camera (Leica Microsystems).

Evaluation and statistical analyses. Immunoreactivity for GSK-3ß was observed in the apical region of glandular formations in tubular adenocarcinoma of the colon, as previously described. Thus, irregular GSK-3ß staining was considered to be significant when $>20 \%$ of cancer cells at the invasive front showed non-apical localization, typically which is known as reversed apical localization. The nuclear B-catenin immunoreactivity at the invasion front was regarded as positive when $>50 \%$ of the nuclei were stained. The correlations between the degree of staining and the subgroups classified according to the clinical and pathological features were calculated by using the Chi-square test at a significance level of $1 \%$.

\section{Results}

Localization of GSK-3ß in normal gut epithelium. As previously shown (12) GSK-3ß was found to be localized in the cytoplasm and on the apical side of simple columnar epithelium. We found that phosphorylated GSK-3ß (Tyr 216), the active form of GSK-3ß, was also localized on the apical side (data not shown). Immunofluorescence data revealed co-localization of GSK- $\beta$ and tight junction-associated protein ZO-1 (Fig. 1).

Localization of GSK-3ß and ZO-1 in stomach cancer. We found that both GSK-3ß and ZO-1 were localized in the apical regions of papillary and tubular adenocarcinoma of the stomach. In signet-ring cell carcinoma, which is considered to be a poorly differentiated phonotype, the expression of GSK-3ß and $\mathrm{ZO}-1$ was reduced and we found dot-like expression in each cell of signet-ring cell carcinoma (Fig. 2).

Localization of GSK-3ß in tubular adenocarcinoma of the colon. Both GSK-3ß and ZO-1 were found to be localized in the apical region of tubular adenocarcinoma of the colon, similar to their localization in normal epithelium; however, the localization of these proteins was different at the invasive front of the cancer. We occasionally found dot-like expression on the basal side of glandular structures in the central area of cancer tissues and the localization of GSK-3B was found to be aberrant in cancer cells at the invasive front. Moreover, reversed apical localization was often observed in cancer cell clusters at the invasive front. In tubular adenocarcinoma of the stomach, the immunoreactivity of GSK-3ß was similar to that observed in tubular adenocarcinoma of the colon (data not shown) (Fig. 3).

Association between aberrant distribution of GSK- $\beta$ at the invasive front and pathological parameters. In 20 out of 

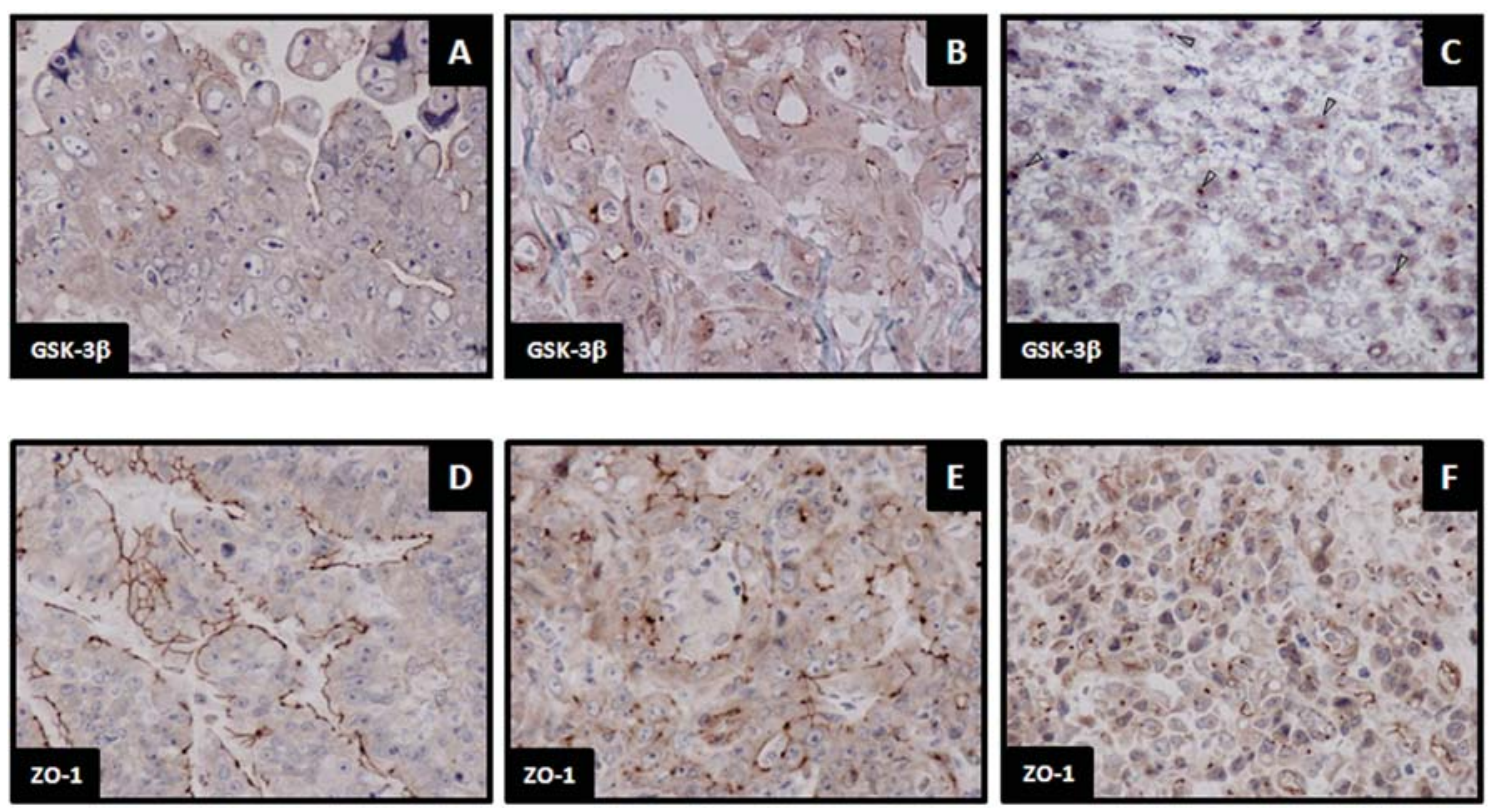

Figure 2. Immunoreactivity of GSK-3B and ZO-1 in stomach cancer. (A and D) Papillary adenocarcinoma. (B and D) Tubular adenocarcinoma. (A-D) Localization of both GSK-3B and ZO-1 localized on the apical side of the glandular structure. (C and E) Signet-ring cell carcinoma. The expression of membrane-localized GSK-3ß and ZO-1 was reduced and dot-like expression was observed in each cell of signet-ring cell carcinoma.
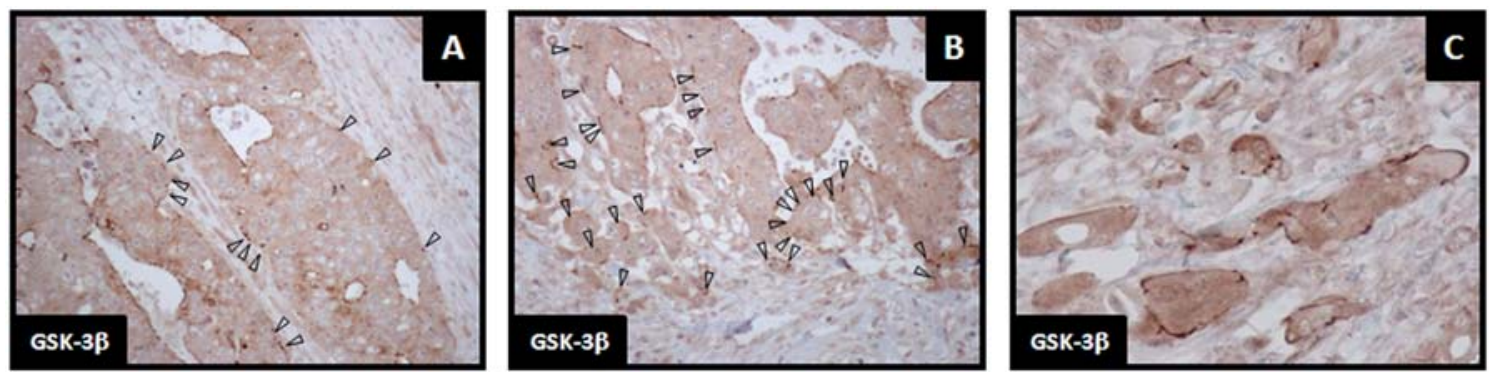

Figure 3. Cellular localization of GSK-3ß in colon cancer. (A) GSK-3ß was localized on the apical side of the glandular formation and occasional dot-like expression on the basal side of the glandular formation. (B and C) Invasive front. GSK-3ß showed remarkable aberrant localization. Note: GSK-3ß was facing towards the extracellular matrix.

40 patients $(50.0 \%)$, the aberrant localization of GSK-3ß at the invasive front was remarkable and was therefore defined as 'severe'. The aberrant localization of GSK-3ß was significantly associated with lymphatic invasion and $\beta$ catenin nuclear accumulation at the invasive front (Table I).

\section{Discussion}

Our immunohistochemical data showed that GSK-3ß and tight junction-associated protein $\mathrm{ZO}-1$ were localized in the same regions in both normal epithelium and cancer cells. In normal simple columnar epithelium, GSK-3ß was localized as a single line at the apical cell border, while in cancer cells, the localization of GSK-3ß had changed. In the case of undifferentiated cancer cells, the expression of both membranelocalized GSK-3ß and ZO-1 was reduced and we found dot-like localization of both proteins in each single cell of signet-ring cell carcinoma. These data suggested that membrane-localized GSK-3ß may be involved in glandular formation of epithelial cells. In tubular adenocarcinoma, we observed apical localization of GSK-3ß in the central lesion
Table I. Association between aberrant localization of GSK-3ß and pathological parameters.

\begin{tabular}{lcc}
\hline & \multicolumn{2}{c}{$\begin{array}{c}\text { Aberrant localization } \\
\text { of GSK-3ß }\end{array}$} \\
\cline { 2 - 3 } & Mild & Severe \\
\hline Total number & 20 & 20 \\
Age $( \pm$ SD) & $77.2 \pm 10.4$ & $78.5 \pm 10.3$ \\
Gender (M/F) & $12 / 8$ & $11 / 9$ \\
Nodal metastasis & 8 & 14 \\
Lymphatic invasion & 8 & $18^{\mathrm{a}}$ \\
Venous invasion & 6 & 12 \\
B-catenin nuclear accumulation & 6 & $19^{\mathrm{a}}$ \\
\hline
\end{tabular}

Aberrant distribution of GSK- $\beta$ was significantly associated to lymphatic invasion and $\beta$-catenin nuclear accumulation at the invasive front $\left({ }^{a} \mathrm{P}<0.01\right)$. 

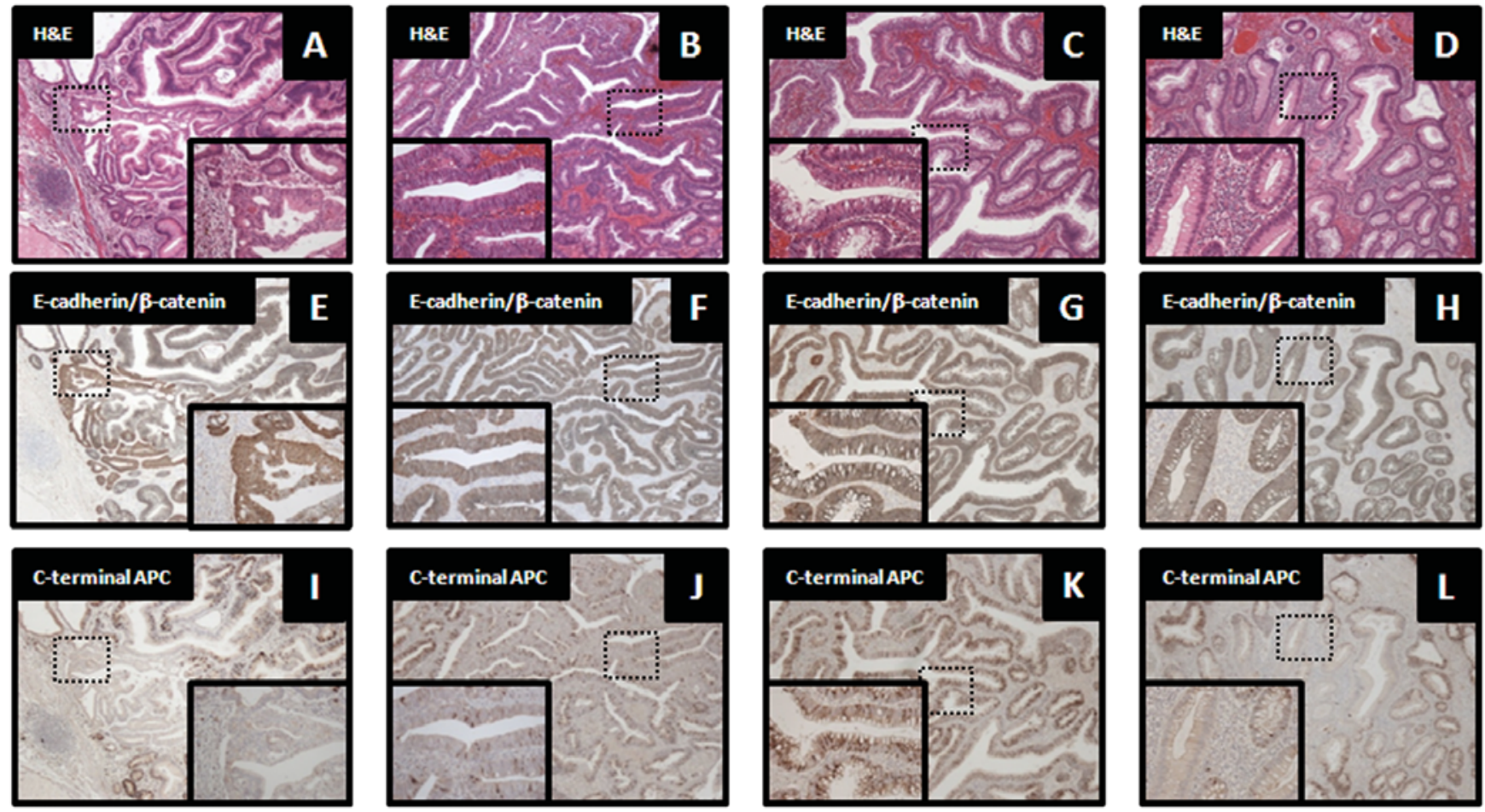

Figure 4. Association of GSK-3ß and APC in adenomatous polyp. (A-D) H\&E staining. (E-H) Double immunostaining for $\beta$-catenin (brown) and E-cadherin (gray). (I-L) Immunostaining for APC. (A, E and I) Serial sections. (A) Infiltrating and/or anchorage-independent cells. (E) B-catenin accumulated in infiltrating and/or anchorage-independent cells. (I) Loss of APC expression. Note: The area wherein the expression of APC was lost was extensive than the area wherein $\beta$-catenin accumulation was observed. (B, F and J) Serial sections. (B) Cells with high-grade nuclear atypia. (F) No B-catenin accumulation in the nucleus. (J) Loss of APC expression. (C, G and K) Serial sections. (C) Cells with moderate-grade nuclear atypia. (F) No B-catenin accumulation in the nucleus. (J) APC expression in the cytoplasm. (D, H and L) Serial sections. (G) Cells with low-grade nuclear atypia. (F) No ß-catenin accumulation in the nucleus. (J) Loss of APC expression.
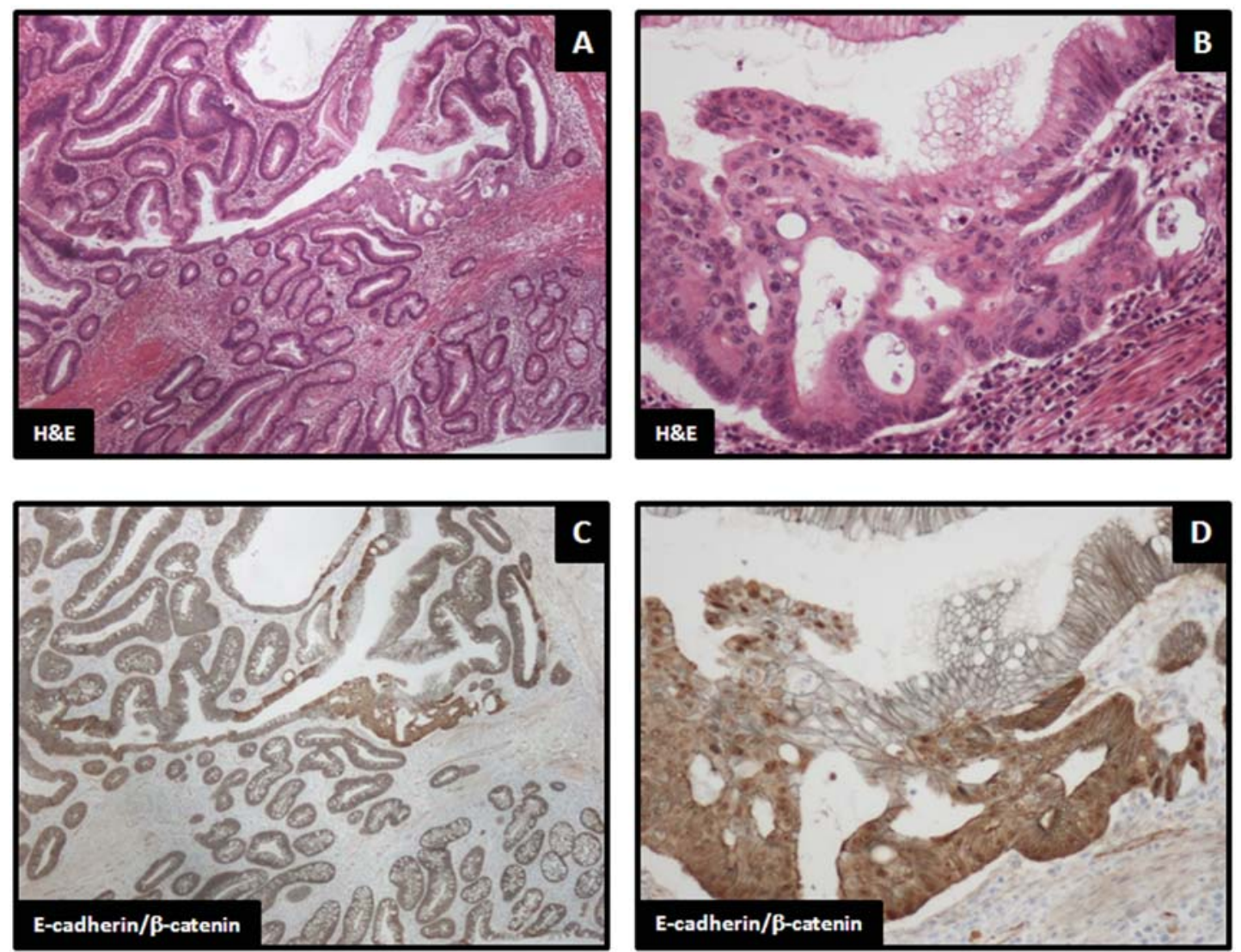

Figure 5. (A and C) Low- and high-magnifications of the double immunostaining for B-catenin (brown) and E-cadherin (gray) in adenomatous polyp. (A and B) Hematoxylin and eosin (H\&E) staining. (C and D) Accumulation of B-catenin in the infiltrating and/or anchorage-independent cells. 
of cancer tissue and aberrant localization of GSK-3ß on the cellular membrane at the invasive front. Our data showed that the aberrant localization of GSK-3ß was associated with lymphatic invasion. We also found that the aberrant localization of GSK-3ß was significantly associated with B-catenin nuclear accumulation at the invasive front (Table I). We thus inferred that the aberrant localization of GSK-3ß may be involved in the accumulation of $\beta$-catenin in the nucleus. Numerous studies showed that the accumulation of B-catenin in the cell nuclei at the invasive front is associated with poor prognosis $(13,14)$. In sporadic and hereditary colorectal cancer, APC gene undergoes $\sim 80-100 \%$ mutation (8). However, the nuclear accumulation of $\beta$-catenin was detected at the invasive front in $\sim 60 \%$ of the cases in our study. These data suggested that APC mutation alone was not sufficient to cause B-catenin nuclear accumulation. Our immunohistochemical data also suggest that a loss or decrease in the expression of APC is necessary but not solely responsible for $\beta$-catenin nuclear accumulation (Fig. 4). Thus, we speculate and our data support that modifying factors such as the tumor architecture or stroma influence the nuclear accumulation of B-catenin (Fig. 5). It has been reported that heparan sulfate proteoglycans, extracellular matrix proteins, regulate the transport of Wnt proteins (15-17). We hypothesize that the aberrant distribution of membrane-related GSK-3ß, that forms unusual interaction with extracellular matrix, is associated with phosphorylation of low-density lipoprotein receptor-related protein (LRP), resulting in the stability of $\beta$-catenin at the invasive front of colorectal cancer $(18,19)$.

In summary, we found that the aberrant localization of GSK-3ß in cancer cells may be associated with malignant behavior of tumor cells. Further investigations are required to clarify the role of GSK-3ß in tumor progression and its polarized localization in colon epithelial cells.

\section{References}

1. Plyte SE, Hughes K, Nikolakaki E, Pulverer BJ and Woodgett JR: Glycogen synthase kinase-3: functions in oncogenesis and development. Biochim Biophys Acta 1114: 147-162, 1992.

2. Cohen P and Frame S: The renaissance of GSK3. Nat Rev Mol Cell Biol 2: 769-776, 2001.

3. Doble BW and Woodgett JR: GSK-3: tricks of the trade for a multi-tasking kinase. J Cell Sci 116: 1175-1186, 2003.

4. Grimes CA and Jope RS: The multifaceted roles of glycogen synthase kinase 3beta in cellular signaling. Prog Neurobiol 65: 391-426, 2001

5. Rubinfeld B, Albert I, Porfiri E, Fiol C, Munemitsu S and Polakis P: Binding of GSK3beta to the APC-beta-catenin complex and regulation of complex assembly. Science 272: 1023-1026, 1996.
6. Dimitriadis A, Vincan E, Mohammed IM, Roczo N, Phillips WA and Baindur-Hudson S: Expression of Wnt genes in human colon cancers. Cancer Lett 166: 185-191, 2001.

7. Smith K, Bui TD, Poulsom R, Kaklamanis L, Williams G and Harris AL: Up-regulation of macrophage wnt gene expression in adenoma-carcinoma progression of human colorectal cancer. Br J Cancer 81: 496-502, 1999.

8. Kinzler KW and Vogelstein B: Lessons from hereditary colorectal cancer. Cell 87: 159-170, 1996.

9. Morin PJ, Sparks AB, Korinek V, Barker N, Clevers H, Vogelstein B and Kinzler KW: Activation of beta-catenin-Tcf signaling in colon cancer by mutations in beta-catenin or APC. Science 275: 1789-1790, 1997.

10. van Es JH, Barker N and Clevers H: You Wnt some, you lose some: oncogenes in the Wnt signaling pathway. Curr Opin Genet Dev 13: 28-33, 2003.

11. Polakis P: The many ways of Wnt in cancer. Curr Opin Genet Dev 17: 45-51, 2007.

12. Hirakawa H, Nakayama T, Shibata K, Mihara Y, Taba M, Nagayasu $\mathrm{T}$ and Sekine I: Apical membrane localization of glycogen synthase kinase $3 \beta$ protein in normal colon epithelium and aberrant distribution in colorectal cancer. Cancer Lett 275: 158-162, 2009.

13. Zhang B, Ougolkov A, Yamashita K, Takahashi Y, Mai M and Minamoto T: Beta-catenin and ras oncogenes detect most human colorectal cancer. Clin Cancer Res 9: 3073-3079, 2003.

14. Ougolkov AV, Yamashita K, Mai M and Minamoto T: Oncogenic beta-catenin and MMP-7 (matrilysin) cosegregate in late-stage clinical colon cancer. Gastroenterology 122: 60-70, 2002.

15. Häcker U, Nybakken K and Perrimon N: Heparan sulphate proteoglycans: the sweet side of development. Nat Rev Mol Cell Biol 67: 530-541, 2005.

16. Lin X: Functions of heparan sulfate proteoglycans in cell signaling during development. Development 131: 6009-6021, 2004.

17. Baeg GH, Lin X, Khare N, Baumgartner S and Perrimon N: Heparan sulfate proteoglycans are critical for the organization of the extracellular distribution of Wingless. Development 128: 87-94, 2001.

18. Zeng X, Tamai K, Doble B, Li S, Huang H, Habas R, Okamura H, Woodgett $\mathrm{J}$ and He X: A dual-kinase mechanism for Wnt coreceptor phosphorylation and activation. Nature 438: 873-877, 2005.

19. Cakigan KM and Liu YI: Wnt signaling: complexity at the surface. J Cell Sci 119: 395-402, 2005. 